\title{
Evaluación en ambiente bioclimático de agroquímicos en la mortandad de los adultos de gorgojo de los Andes (Premnotrypes spp.) en papa
}

\author{
Evaluation in a bioclimatic environment of agrochemicals \\ in the mortality of adults of the potato Andean weevils (Premnotrypes spp.)
}

\author{
aLindo Gutarra, E. \\ Facultad de Agronomía / Universidad Nacional del Centro del Perú \\ Email: elindo@uncp.edu.pe
}

\section{Resumen}

Los adultos de gorgojo de los Andes (Premnotrypes spp.); fueron sometidos a estudios de los efectos de los ingredientes activos clotianidin, fipronil, tiametoxam, metaflubizona, carbofuran, clorpirifos y lambdacialotrina; para ello, se instaló un ambiente bioclimático, donde se usó 10 adultos por frasco de $500 \mathrm{cc}$ de capacidad y se sometió las hojas tratadas en el tercer, séptimo y décimo cuarto día. Se evaluó el número de adultos vivos en el primer, cuarto y décimo cuarto día. Los datos registrados fueron interpretados en base a histogramas, los análisis estadísticos fueron contrastados en base al diseño completamente al azar con 4 repeticiones y el test de Tukey a un $95 \%$ de confiabilidad y, para la eficiencia, se utilizó la fórmula de Abbot. Se arrivaron a los siguientes resultados; al tercer día de control, los tratamientos que sobresalieron fueron: Dantotsu (Clotianidin, $200 \mathrm{~g} / 200 \mathrm{l}$ de agua), Murder (Fipronil y tiametoxam, 300 cc/ $200 \mathrm{l}$ de agua) y Regent (Fipronil, 300 cc/ $200 \mathrm{l}$ de agua) registrándose 2,00 a 2,75 adultos vivos promedio, al séptimo día del control los tratamientos que sobresalieron fueron: Dantotsu (Clotianidin, 200 g/ 200 l de agua) y Murder (Fipronil y tiame-toxam, $300 \mathrm{cc} /$ 2001 de agua), registrándose 0,75 a 1,25 adultos vivos promedio; al décimo cuarto día, sobresalieron Dantotsu (Clotianidin, $200 \mathrm{~g} / 200 \mathrm{l}$ de agua, Murder (Fipronil y tiametoxam, $300 \mathrm{cc} /$ $200 \mathrm{l}$ de agua) y Regent (Fipronil, 300 cc/ $200 \mathrm{l}$ de agua), se registró 2,50 a 3,00 adultos vivos promedio; al décimo cuarto día de control, los tratamientos que sobresalieron fueron: Dantotsu (Clotianidin, 200 g/ $200 \mathrm{l}$ de agua), Murder (Fipronil y tiametoxam, $300 \mathrm{cc} / 200 \mathrm{l}$ de agua) y Regent (Fipronil, $300 \mathrm{cc} / 200 \mathrm{l}$ de agua), no se registró adultos vivos al cuarto día de evaluación.

La mayor eficiencia fue registrada en Dantotsu (Clotianidin, 200 g/ 200 l de agua) con una eficiencia del $100 \%$ hasta los 14 días después de aplicar los tratamientos, seguido de Murder 300 cc/200 l de agua con una eficiencia de $100 \%$ hasta los 7 días después del control y $90 \%$ en el décimocuarto día.

Palabras clave: Premnotrypes spp., bioclimático, papa, agroquímico

\begin{abstract}
Adults of the Andes weevil (Premnotrypes spp.) were subjected to the studies of the effects of the active ingredients Clotianidin, Fipronil, thiamethoxam, Metaflubizona, Carbofuran, Chlorpyrifos and Lambdacialothrin; accordinaly, a bioclimatic environment was installed, where 10 adults per 500 cc capacity bottle and the treated leaves were subjected on the third, seventh and fourteenth days. The number of living adults was evaluated on the first day, fourth and fourteenth. The recorded data were interpreted based on histograms and the statistical analyzes were contrasted on the basis of the completely randomized design with 4 repetitions the Tukey test at $95 \%$ reliability, and the Abbot formula was used for efficiency. The following results were obtained; on the third control day, the treatments that stood out were: Dantotsu (Clotianidin, 200 g / $200 \mathrm{l}$ of water), Murder (Fipronil and thiamethoxam, 300cc / $200 \mathrm{l}$ of water) and Regent (Fipronil, 300cc / $200 \mathrm{l}$ of water) registering 2.00 to 2.75 average live adults, on the seventh day of the control the treatments that stood out were: Dantotsu (Clotianidin, $200 \mathrm{~g} / 200 \mathrm{l}$ of water) and Murder (Fipronil and thiamethoxam, $300 \mathrm{cc} / 200 \mathrm{l}$ of water) registering 0.75 to 1.25 average live adults, on the fourteenth day the following stood out: Dantotsu (Clotianidin, $200 \mathrm{~g} / 200 \mathrm{l}$ of water, Murder (Fipronil and thiamethoxam, $300 \mathrm{cc} / 200$ l of water) and Regent (Fipronil, 300 cc / 200 l of water). Registering no living adults on the fourth evaluation day.

The highest efficency was registerd in Dantotsu (Clotianidin, $200 \mathrm{~g} / 200 \mathrm{l}$ of water) with an $100 \%$ efficiency up to 14 days after applying the treatments, followed by Murder $300 \mathrm{cc} / 200 \mathrm{l}$ of water with an efficiency of $100 \%$ up to 7 days after control and $90 \%$ on the fourteenth day.
\end{abstract}

Keywords: Premnotrypes spp., bioclimatic, potato, agrochemical 


\section{Introducción}

El cultivo de papa en el valle del Mantaro es apreciado por el campesino andino, principalmente, porque es una alimento básico en sus mesas; además, le reporta utilidades importantes para su economía. Sin embargo, años tras año el cultivo enfrenta a un sin número de problemas de plagas, enfermedades, malezas, etc., donde el agricultor enfrenta estos problemas con el sustento del control químico para lograr una cosecha aparentemente razonable, sin considerar, muchas veces, los efectos colaterales que puede estar ocasionando a los que consumen el tubérculo. Asimismo, se debe mencionar el problema fitosanitario de mayor importancia, es el complejo gorgojo de los andes y, para contrarrestar el efecto de sus daños, se usa una serie a insecticidas de diversos grupos químicos, que relativamente tienen efectos similares respecto al control de los adultos de esta plaga.

Con el objetivo de visualizar con mayor precisión sus efectos, es necesario evaluarlo, controlando la población de adultos bajo un ambiente bioclimático. Bajo estas características, se planteó los siguientes objetivos: Determinar los efectos de los agroquímicos frecuentemente usados por los agricultores en la mortandad de los adultos de gorgojo de los Andes. Con los objetivos específicos siguientes: (1) Determinar los efectos de los carbamatos y fosforados en la mortandad de los adultos del gorgojo de los Andes; (2) Determinar los efectos de los neonicotinoides y metaflubizona en la mortandad de los adultos del gorgojo de los Andes y; (3) Comparar la eficiencia de las mezclas en la mortandad de los adultos del gorgojo de los Andes.

\section{Métodos y materiales}

El presente trabajo se realizó en ambientes bioclimáticos del distrito de Sicaya en Huancayo - Perú.

\section{Lugar de ejecución}

Ubicación temporal: El presente experimento fue conducido durante la campaña agrícola 2018-2019, desde noviembre de 2018 hasta junio de 2019 en un ambiente bioclimático.

Ubicación política: Departamento de Junín, provincia de Huancayo y distrito de Sicaya

Ubicación geográfica: Altitud: $3273 \mathrm{msnm}$, Latitud: $12^{\circ} 00$ ' 45” y Longitud: 75¹7'00”, a $12^{\circ} 02^{\prime} 18^{\prime}$ " del Ecuador latitud sur y $75^{\circ} 18^{\prime} 24^{\prime \prime}$ del meridiano de Greenwich longitud oeste; al norte de la ciudad de Huancayo a una distancia de $12 \mathrm{~km}$.

\section{Metodología}

\section{Aplicaciones de los tratamientos}

Los tratamientos se aplicaron en plantas emergidas y se marcaron por tratamiento. De estas plantas, se extrajeron foliolos para darles de alimento a los adultos del gorgojo a los 3 días después de aplicar los tratamientos (DDALT), a los 7 (DDALT), a los 14 (DDALT) y, 18 (DDALT). Por otro lado, después de aplicar los tratamientos, se evaluaron del primer hasta el cuarto día en tinglado.

\section{Recolección de adultos}

Los adultos, se colectaron de campos infestados en número suficientes para evaluar los tratamientos. Estos adultos se mantuvieron con alimentación de foliolos sanos hasta someterlos a los tratamientos.

\section{Vasos de $500 \mathrm{cc}$}

En frascos de $500 \mathrm{cc}$, se ubicaron los foliolos tratados donde se ubicaron a 10 adultos vivos de gorgojo de los Andes por frasco, que correspondió a un tratamiento y una repetición.

\section{Evaluaciones}

Se evaluó, después del control, al 3, 7 y 14 días. Luego se observó la mortandad del primer al cuarto día después de habérsele alimentado con el foliolo tratado, para registrar adultos vivos y muertos.

\section{Tratamientos}

\section{T0: Testigo absoluto}

T1: Verismo $500 \mathrm{cc} / 200 \mathrm{l}$ de agua

T2: Furadan $1000 \mathrm{cc} / 200 \mathrm{l}$ de agua

T3: Tifon $500 \mathrm{cc} / 200 \mathrm{l}$ de agua

T4: Dantotsu 200 cc/200 l de agua

T5: Regent $300 \mathrm{cc} / 200 \mathrm{l}$ de agua

T6: Engeo 200/200 l de agua

T7: Murder 300 cc/200 l de agua

Para determinar el producto de mayor eficiencia, se utilizó el diseño completamente al azar. Se aplicaron 3 respeticiones a los 8 tratamientos.

\section{Determinación de la eficiencia relativa}

Para determinar la eficiencia de los insecticidas, se utilizó la fórmula de Abott.

$$
\% E=\left[1-\frac{T d}{C d}\right] \times 100
$$

Donde: $\% E$ es el porcentaje de eficiencia, $T d$ es la infestación en parcela (Frasco de foliolos tratados) después de aplicar el tratamiento y Cd es la infestación en parcela (Frasco de foliolos tratados) testigo después de aplicar el tratamiento.

\section{Población y muestra}

La población, estuvo conformada por todos los adultos de gorgojo de los Andes que se recolectaron de los campos infestados. La muestra representa a todos los adultos de Premnotrypes spp. que se encuentran en el área experimental.

Para determinar la mortandad de adultos, se contabilizó el número de adultos vivos y muertos en cada frasco después del control. 


\section{Resultados y discusión}

\section{Adultos vivos al tercer día del control}

En la Tabla 1, del análisis de ANVA, Se observan los resultados de las hojas ingeridas al tercer día después del control y de la evaluación al primer día. Se observa una alta significación estadística que conlleva a concluir que, entre los tratamientos existe una diferencia sustancial en relación a los adultos vivos. El coeficiente de determinación fue del $98 \%$ que atribuye que los tratamientos tuvieron efectos en esa proporción y un $2 \%$ a factores que no fueron controlados en el experimento bioclimático.

\section{Tabla 1}

Evaluación del efecto de insecticidas sobre adultos de Prenotrypes spp. en hojas de papa al tercer día de aplicación y primer día de evaluación. 2019

\begin{tabular}{lcccc}
\hline \multirow{2}{*}{$\begin{array}{c}\text { Fuentes de } \\
\text { variabilidad }\end{array}$} & $\begin{array}{c}\text { Grados } \\
\text { de } \\
\text { libertad }\end{array}$ & \multicolumn{3}{c}{ Tercer día de aplicación } \\
\cline { 3 - 5 } & $\begin{array}{c}\text { Cuadrados } \\
\text { medios }\end{array}$ & $\begin{array}{c}\text { Prueba } \\
\text { de F }\end{array}$ & Sign. \\
\hline Tratamientos & 7 & 28,86 & 184,16 & 0,01 \\
Error & 24 & 0,15 & & \\
Total & 31 & & & \\
Promedio & & 4,63 & \\
$\begin{array}{l}\text { Coeficiente } \\
\text { determinación }\end{array}$ & & 0,98 & \\
CV (\%) & & 8,26 & \\
\hline
\end{tabular}

En la Tabla 2, se observa que en el testigo no hubo mortandad, debido a que se encontró un promedio de 10 adultos vivos, en el tratamiento Dantotsu (200 g/ $200 \mathrm{l}$ de agua) se registró 2,0 adultos vivos promedio, que fue estadísticamente similar a Murder y Regent que reportaron 2,25 a 2,75 adultos vivos promedio, significa tuvieron mayor efectividad que el resto de los tratamientos. Cabe señalar que Dantotsu es el clothianidin, un neonicotinoide de tercera generación; asimismo, Regent es un fenil pirazol que tiene un buen efecto coleoptericida; mientras, Murder es una mezcla de un neonicotinoide con un fenil pirazol, es decir, estas nuevas moléculas en la actualidad, tienen un efecto importante en el control inmediato de adultos de gorgojo de los Andes, que en parte serviría como un componente de mayor jerarquía para el manejo integrado de plagas para el cultivo de papa en el valle del Mantaro.

Para mayor visualización, en la Figura 1 se representan en el histograma, el promedio de adultos vivos de los 8 tratamientos, en el testigo se mantuvo un promedio de 10 adultos vivos; es decir, que ningún individuo murió en las 4 repeticiones. Asimismo, se observa, tanto el tifón (fosforado) como el Furadan (carbamato), que su efecto en la mortandad de adultos no sobrepasa el 50 $\%$; es decir, su efecto es relativamente insuficiente para controles inmediatos.

Tabla 2

Prueba de Tukey. Confiabilidad de

0,05 para el tercer día de

aplicación y primer día de evaluación

\begin{tabular}{lccc}
\hline \multicolumn{1}{c}{ Tratamiento } & & \\
\cline { 1 - 2 } Producto & $\begin{array}{c}\text { Dosis } \\
\text { (200 I agua) }\end{array}$ & Promedio & Significación \\
T5 Dantotsu & $200 \mathrm{~g}$ & 2,00 & $\mathrm{~A}$ \\
T8 Murder & $300 \mathrm{cc}$ & 2,25 & $\mathrm{~A}$ \\
T6 Regent & $300 \mathrm{cc}$ & 2,75 & $\mathrm{~A}$ \\
T7 Engeo & $200 \mathrm{cc}$ & 4,50 & $\mathrm{~B}$ \\
T4 Tifón & $500 \mathrm{cc}$ & 4,50 & $\mathrm{~B}$ \\
T3 Furadan & $1000 \mathrm{cc}$ & 5,00 & $\mathrm{~B}$ \\
T2 Verismo & 500 & 6,00 & $\mathrm{C}$ \\
T1 Testigo & & 10,00 & $\mathrm{C}$ \\
\hline
\end{tabular}

Figura 1

Acción de insecticidas en el control de

Premnotrypes spp. en el cultivo de papa,

al primer día de aplicación y primer

día de evaluación. Huancayo - 2019

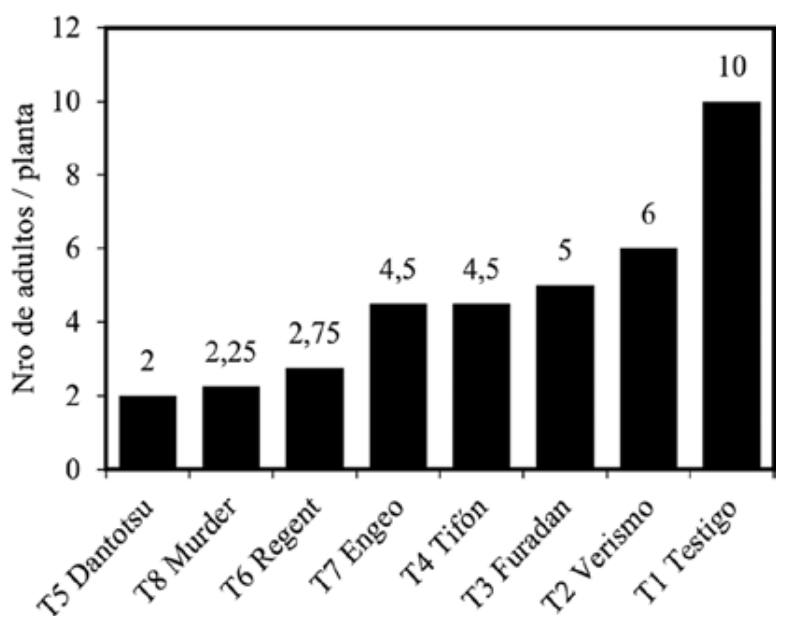

\section{Adultos vivos al séptimo día de control}

En la Tabla 3, del análisis de ANVA, se observan los resultados de las hojas ingeridas al séptimo día, después del control y de la evaluación al primer día. Del análisis de variancia, se observa una alta significación estadística, que conlleva a concluir que entre los tratamientos existe una diferencia sustancial en relación a los adultos vivos. El coeficiente de determinación fue del $99 \%$ que atribuye que los tratamientos tuvieron efectos en esa proporción y un $1 \%$ a factores que no fueron controlados en el experimento bioclimático. 
Tabla 3

Evaluación del efecto de insecticidas sobre adultos de Prenotrypes spp. En hojas de papa al tercer día de aplicación y primer día de evaluación. 2019

\begin{tabular}{lcccc}
\hline \multirow{2}{*}{$\begin{array}{c}\text { Fuentes de } \\
\text { variabilidad }\end{array}$} & $\begin{array}{c}\text { Grados } \\
\text { de } \\
\text { libertad }\end{array}$ & \multicolumn{3}{c}{ Tercer día de aplicación } \\
\cline { 3 - 5 } & $\begin{array}{c}\text { Cuadrados } \\
\text { medios }\end{array}$ & $\begin{array}{c}\text { Prueba } \\
\text { de F }\end{array}$ & Sign. \\
\hline Tratamientos & 7 & 37,34 & 358,46 & 0,01 \\
Error & 24 & 0,10 & & \\
Total & 31 & & & \\
Promedio & & 4,06 & & \\
$\begin{array}{l}\text { Coeficiente } \\
\text { determinación }\end{array}$ & & 0,99 & & \\
CV (\%) & & 7,94 & & \\
\hline
\end{tabular}

En la Tabla 4, que representan la prueba de Tukey se observa que en el testigo no hubo mortandad, debido a que se encontró un promedio de 10 adultos vivos, en el tratamiento Dantotsu (200 g/ 200 l de agua) se registró 0,75 adultos vivos promedio que fue estadísticamente similar a Murder que se registró 1,25 individuos vivos y esta, a la vez, fue similar a Regent que reportó 2,0 adultos vivos promedio, que significa que después de habérsele aplicado a 7 días y evaluarlos al primer día es un resultado satisfactorio; es decir, el efecto de estos tres productos comerciales se intensifica con una mejor mortandad de adultos; sin embargo, tanto los fosforado, carbamatos y Metaflubizona no tuvieron un buen efecto en el control de adultos de gorgojo de los Andes. Esta respuesta se debe básicamente a que los fosforados y carbamatos se usaron más de 30 años y es probable que exista resistencia de parte de la plaga.

Tabla 4

Prueba de Tukey. Confiabilidad de 0,05 para el tercer día de aplicación y primer día de evaluación

\begin{tabular}{lccc}
\hline \multicolumn{2}{c}{ Tratamiento } & & \\
Producto & $\begin{array}{c}\text { Dosis } \\
\text { (200 I agua) }\end{array}$ & Promedio & Significación \\
\hline T5 Dantotsu & $200 \mathrm{~g}$ & 0,75 & $\mathrm{~A}$ \\
T8 Murder & $300 \mathrm{cc}$ & 1,25 & $\mathrm{~A} \mathrm{~B}$ \\
T6 Regent & $300 \mathrm{cc}$ & 2,00 & $\mathrm{~B}$ \\
T7 Engeo & $200 \mathrm{cc}$ & 3,00 & $\mathrm{C}$ \\
T4 Tifón & $500 \mathrm{cc}$ & 4,00 & $\mathrm{D}$ \\
T3 Furadan & $1000 \mathrm{cc}$ & 5,50 & $\mathrm{E}$ \\
T2 Verismo & 500 & 6,00 & $\mathrm{E}$ \\
T1 Testigo & & 10,00 & $\mathrm{~F}$ \\
\hline
\end{tabular}

Para mayor visualización, la Figura 2 representa en el histograma el promedio de adultos vivos de los 8 tratamientos, el testigo mantuvo un promedio de 10 adultos vivos; es decir, que ningún individuo murió en las 4 repeticiones. Asimismo, se observan, tanto el tifón (fosforado) como el Furadan (carbamato) entre 4 a 5,5 adultos vivos; es decir, su efecto en la mortandad de adultos no sobrepasa el $50 \%$, esta respuesta respecto a la tendencia fue similar al tercer día. Respecto a la muestra, en ese caso fue menor.

\section{Figura 2}

Acción de insecticidas en el control de Premnotrypes spp. en el cultivo de papa, al séptimo día de aplicación y primer día de evaluación. Huancayo - 2019

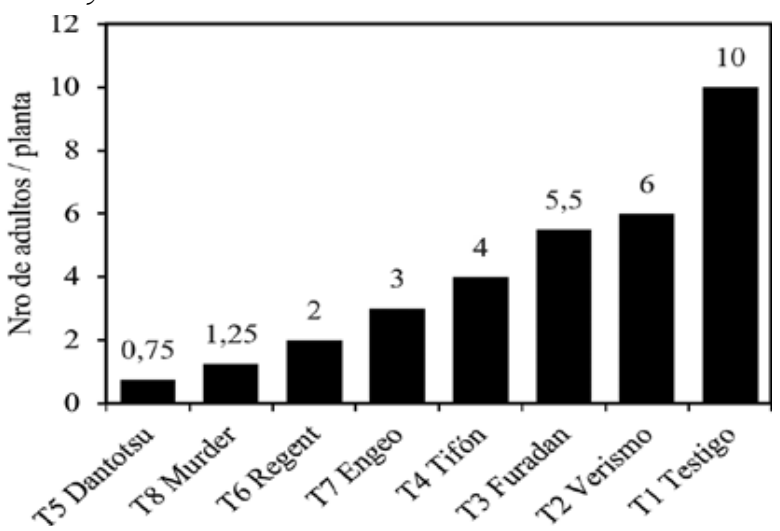

\section{Adultos vivos al día 14 del control y primer día de evaluación}

En la Tabla 5, del análisis de ANOVA, Se observan los resultados de las hojas ingeridas al séptimo día después del control y de la evaluación al primer día. Del análisis de variancia, se observa una alta significación estadística que conlleva a concluir que entre los tratamientos existe una diferencia sustancial en relación a los adultos vivos. El coeficiente de determinación fue del $99 \%$ que atribuye que los tratamientos tuvieron efectos en esa proporción y un $1 \%$ a factores que no fueron controlados en el experimento bioclimático.

\section{Tabla 5}

Evaluación del efecto de insecticidas sobre adultos de Premnotrypes spp. En hojas de papa al día 14 de aplicación y primer día de evaluación. 2019

\begin{tabular}{|c|c|c|c|c|}
\hline \multirow{2}{*}{$\begin{array}{c}\text { Fuentes de } \\
\text { variabilidad }\end{array}$} & \multirow{2}{*}{$\begin{array}{c}\text { Grados de } \\
\text { libertad }\end{array}$} & \multicolumn{3}{|c|}{ Tercer día de aplicación } \\
\hline & & $\begin{array}{l}\text { Cuadrados } \\
\text { medios }\end{array}$ & $\begin{array}{c}\text { Prueba } \\
\text { de F }\end{array}$ & Sign. \\
\hline Tratamientos & 7 & 25,92 & 226,25 & 0,01 \\
\hline Error & 24 & 0,11 & & \\
\hline Total & 31 & & & \\
\hline Promedio & & 4,84 & & \\
\hline $\begin{array}{l}\text { Coeficiente } \\
\text { determinación }\end{array}$ & & 0,99 & & \\
\hline $\mathrm{CV}(\%)$ & & 6,99 & & \\
\hline
\end{tabular}

En la Tabla 6, que representan la prueba de Tukey, se observa que en el testigo no hubo mortandad, debido a que se encontró un promedio de 10 adultos vivos, en el tratamiento Dantotsu (200 g/ 200 l de agua) se registró 2,50 adultos vivos promedio que fue estadísticamente similar a Murder y Regent, que se registraron 2,75 y 3,00 
individuos vivos y Tifon y Engeo estadísticamente fueron similares y se registró entre 4 a 4,5 adultos vivos, esta tendencia de mortandad de adultos de gorgojo de los Andes se mantiene desde los tres días después de habérsele aplicado a las hojas de papa con ciertas diferencias numéricas.

Tabla 6

Prueba de Tukey. Confiabilidad de 0,05 para el día 14 de aplicación y primer día de evaluación

\begin{tabular}{lccc}
\hline \multicolumn{1}{c}{ Tratamiento } & & \\
\cline { 1 - 2 } Producto & $\begin{array}{c}\text { Dosis } \\
\text { (200 l agua) }\end{array}$ & Promedio & Significación \\
T5 Dantotsu & $200 \mathrm{~g}$ & 2,50 & $\mathrm{~A}$ \\
T8 Murder & $300 \mathrm{cc}$ & 2,75 & $\mathrm{~A}$ \\
T6 Regent & $300 \mathrm{cc}$ & 3,00 & $\mathrm{~A}$ \\
T7 Engeo & $200 \mathrm{cc}$ & 4,00 & $\mathrm{~B}$ \\
T4 Tifón & $500 \mathrm{cc}$ & 4,50 & B C \\
T3 Furadan & $1000 \mathrm{cc}$ & 5,00 & $\mathrm{C}$ \\
T2 Verismo & 500 & 7,00 & D \\
T1 Testigo & & 10,00 & $\mathrm{E}$ \\
\hline
\end{tabular}

En la Figura 3, representan en el histograma el promedio de adultos vivos de los 8 tratamientos, el testigo mantuvo un promedio de 10 adultos vivos; es decir, que ningún individuo murió en las 4 repeticiones; asimismo, se observan, tanto el dantotsu (Clothianadin) como el regent (fenil pirazol), una población menor de adultos vivos donde se reportó entre 2,5 a 2,75 individuos respectivamente, esta respuesta asevera la tendencia que ocurrió a los 3 y 7 días después del control.

\section{Figura 3}

Acción de insecticidas en el control de Premnotrypes spp. en el cultivo de papa, al día 14 de aplicación y primer día de evaluación. Huancayo - 2019

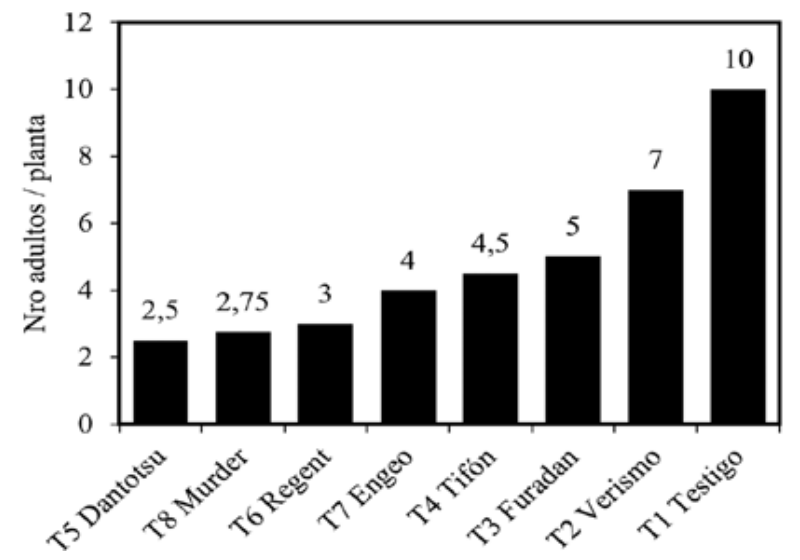

Adultos vivos al día 14 del control y cuarto día de evaluación

En la Tabla 7, del análisis de varianza, se observan los resultados de las hojas ingeridas al décimocuarto día después del control y de la evaluación al cuarto día. Del análisis de varianza se observa una alta significación estadística que conlleva a concluir que entre los tratamientos existe una diferencia sustancial en relación a los adultos vivos. El coeficiente de determinación fue del $93 \%$ que atribuye que los tratamientos tuvieron efectos en esa proporción y un $7 \%$ a factores que no fueron controlados en el experimento bioclimático. Por otro lado, se observa un coeficiente de variabilidad alta que corresponde a un $41,13 \%$ que se explica que dentro de cada tratamiento hubo una alta heteregeonidad.

\section{Tabla 7}

Evaluación del efecto de insecticidas sobre adultos de Premnotrypes sp. En hojas de papa al día 14 de aplicación y cuarto día de evaluación. 2019

\begin{tabular}{lcccc}
\hline \multirow{2}{*}{$\begin{array}{c}\text { Fuentes de } \\
\text { variabilidad }\end{array}$} & Grados de & \multicolumn{3}{c}{ Tercer día de aplicación } \\
\cline { 3 - 5 } & libertad & $\begin{array}{c}\text { Cuadrados } \\
\text { medios }\end{array}$ & $\begin{array}{c}\text { Prueba } \\
\text { de F }\end{array}$ & Sign. \\
\hline Tratamientos & 7 & 48,17 & 46,71 & 0,01 \\
Error & 24 & 1,03 & & \\
Total & 31 & & & \\
Promedio & & 2,47 & & \\
Coeficiente & & 0,93 & \\
determinación & & & \\
CV (\%) & & 41,13 & \\
\hline
\end{tabular}

En la Tabla 8, que representa la prueba de Tukey, se observan que los tratamientos Dantotsu (200 g/ $200 \mathrm{l} \mathrm{de}$ agua) a Murder (300 cc) y Regent (300 cc) no se registraron adultos vivos y que estadísticamente fue similar a tratamiento con Furadan, Engeo y Tifon. En estos casos hubo en promedio de 1 a 2 individuos vivos, que en la vida práctica de campo es un problema grave cuando se tienen al menos un individuo vivo por la capacidad de reproducción que va repercutir daños graves en los tubérculos de papa. Por otro lado, el verismo no tuvo un buen efecto en el control de adultos de gorgojo de los Andes a las dosis que se aplicó, observándose en promedio 5 adultos vivos.

\section{Tabla 8}

Prueba de Tukey. Confiabilidad de 0,05 para el día 14 de aplicación y cuarto día de evaluación

\begin{tabular}{lccc}
\hline \multicolumn{1}{c}{ Tratamiento } & & \\
\cline { 1 - 2 } Producto & $\begin{array}{c}\text { Dosis } \\
\text { (200 I agua) }\end{array}$ & Promedio & Significación \\
T5 Dantotsu & $200 \mathrm{~g}$ & 0,00 & $\mathrm{~A}$ \\
T8 Murder & $300 \mathrm{cc}$ & 0,00 & $\mathrm{~A}$ \\
T6 Regent & $300 \mathrm{cc}$ & 0,00 & $\mathrm{~A}$ \\
T7 Engeo & $200 \mathrm{cc}$ & 1,00 & $\mathrm{~A}$ \\
T4 Tifón & $500 \mathrm{cc}$ & 1,75 & $\mathrm{~A}$ \\
T3 Furadan & $1000 \mathrm{cc}$ & 2,00 & $\mathrm{~A}$ \\
T2 Verismo & 500 & 5,00 & $\mathrm{~B}$ \\
T1 Testigo & & 10,00 & $\mathrm{C}$ \\
\hline
\end{tabular}


En la Figura 4, se representa en el histograma el promedio de adultos vivos de los 8 tratamientos, los tratamientos Dantotsu (Clothianadin), como el Regent (Fenil pirazol) y murder controlaron un $100 \%$ en esta etapa, que en la práctica, representa una alternativa importante para controlar adultos de gorgojo de los Andes; mientras los fosforados, carbamatos y el verismo no tienen una buena capacidad para el control de esta plaga.

\section{Figura 4}

Acción de insecticidas en el control de Premnotrypes spp. en el cultivo de papa, al día 14 de aplicación y

cuarto día de evaluación. Huancayo - 2019

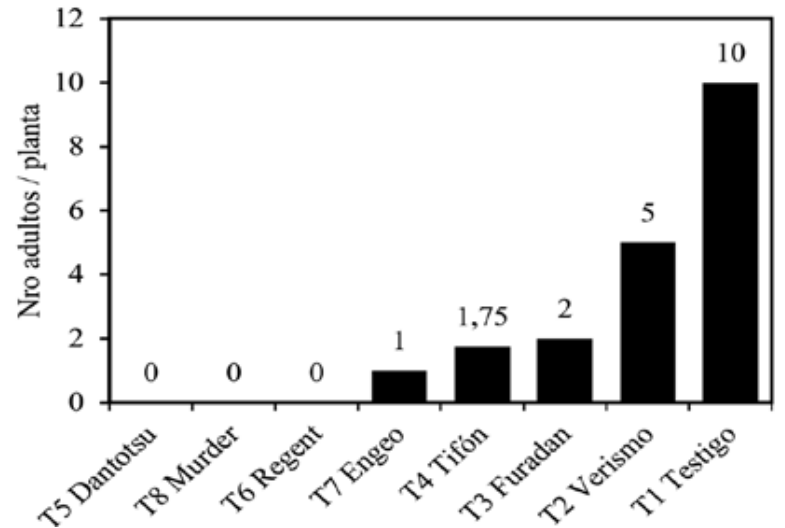

Tabla 9

$\%$ de eficiencia de los tratamientos al tercer día de evaluación en los cuatro momentos (días 3, 7, 14 y 21) al someter a los adultos de gorgojo de los Andes

\begin{tabular}{|c|c|c|c|c|c|c|c|c|}
\hline \multirow{2}{*}{ Tratamientos } & \multicolumn{2}{|c|}{ Día 3} & \multicolumn{2}{|c|}{ Día 7} & \multicolumn{2}{|c|}{ Día 14} & \multicolumn{2}{|c|}{ Día 18} \\
\hline & $\% \mathbf{E}$ & P\%E & $\% \mathbf{E}$ & P\%E & $\% \mathbf{E}$ & P\%E & $\% \mathbf{E}$ & P\%E \\
\hline \multirow{4}{*}{ Testigo } & 0 & \multirow{4}{*}{0} & 0 & \multirow{4}{*}{0} & 0 & \multirow{4}{*}{0} & 0 & \multirow{4}{*}{0} \\
\hline & 0 & & 0 & & 0 & & 0 & \\
\hline & 0 & & 0 & & 0 & & 0 & \\
\hline & 0 & & 0 & & 0 & & 0 & \\
\hline \multirow{4}{*}{$\begin{array}{l}\text { Verismo } \\
5 \mathrm{cc} / 200 \mathrm{~L}\end{array}$} & 90 & \multirow{4}{*}{90} & 90 & \multirow{4}{*}{85} & 80 & \multirow{4}{*}{77,5} & 50 & \multirow{4}{*}{50} \\
\hline & 80 & & 80 & & 80 & & 50 & \\
\hline & 90 & & 90 & & 70 & & 50 & \\
\hline & 100 & & 80 & & 80 & & 50 & \\
\hline \multirow{4}{*}{ Furadan $1000 \mathrm{cc} / 200$ litros } & 100 & \multirow{4}{*}{90} & 100 & \multirow{4}{*}{95} & 80 & \multirow{4}{*}{80} & 60 & \multirow{4}{*}{57,5} \\
\hline & 90 & & 100 & & 80 & & 60 & \\
\hline & 80 & & 90 & & 80 & & 60 & \\
\hline & 90 & & 90 & & 80 & & 50 & \\
\hline \multirow{4}{*}{$\begin{array}{l}\text { Tifon } \\
500 \mathrm{cc} / 200 \text { litros }\end{array}$} & 100 & \multirow{4}{*}{92,5} & 100 & \multirow{4}{*}{100} & 90 & \multirow{4}{*}{90} & 50 & \multirow{4}{*}{60} \\
\hline & 90 & & 100 & & 100 & & 60 & \\
\hline & 80 & & 100 & & 80 & & 60 & \\
\hline & 100 & & 100 & & 90 & & 70 & \\
\hline \multirow{4}{*}{ Dantotsu $200 \mathrm{~g} / 200$ litros } & 100 & \multirow{4}{*}{100} & 100 & \multirow{4}{*}{100} & 100 & & 50 & \\
\hline & 100 & & 100 & & 100 & 100 & 60 & 575 \\
\hline & 100 & & 100 & & 100 & 100 & 60 & נו, דו \\
\hline & 100 & & 100 & & 100 & & 60 & \\
\hline & 100 & & 100 & & 90 & & 50 & \\
\hline Pooent 200 oo/200 litro & 90 & 100 & 100 & 100 & 100 & 00 & 60 & 60 \\
\hline 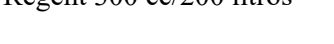 & 90 & & 100 & & 80 & & 60 & \\
\hline & 100 & & 100 & & 90 & & 70 & \\
\hline & 90 & & 70 & & 70 & & 50 & \\
\hline Fngeo $200 \mathrm{cc} / 200$ litros & 90 & 05 & 80 & 825 & 70 & 77 & 60 & \\
\hline Lingeo $2000 \mathrm{c} / 200$ intios & 80 & 95 & 80 & $82, J$ & 80 & 1/,5 & 60 & 60 \\
\hline & 100 & & 100 & & 90 & & 70 & \\
\hline & 100 & & 100 & & 90 & & 70 & \\
\hline L 200 - & 100 & 100 & 100 & 100 & 100 & $0 \Omega$ & 70 & 0 \\
\hline nurder sou cc/ 200 iltos & 100 & 100 & 100 & 100 & 80 & 90 & 70 & 10 \\
\hline & 100 & & 100 & & 90 & & 70 & \\
\hline
\end{tabular}




\section{Eficiencia de los tratamientos}

En la Tabla 9, se registra el porcentaje de eficiencia de los tratamientos al tercer día de evaluación cuando fueron sometidos los adultos de gorgojo de los Andes al tercer, séptimo, décimo cuarto y décimo octavo día de aplicar los tratamientos. Se observa en Dantotsu (200 cc / 200 l de agua) una eficiencia del 100\% hasta los 14 días después de aplicar los tratamientos; seguido de Murder (300 cc/200 l de agua) una eficiencia de $100 \%$ hasta los 7 días después del control y $90 \%$ en 14 días y; el tratamiento con Regent, es de similar al del Murder, registrándose de 90 a $100 \%$ de eficiencia hasta los 14 días después del control. Los tratamientos con carbamatos, fosforados no logran un porcentaje óptimo.

\section{Conclusiones}

- Al día 3 del control, los tratamientos que sobresalieron fueron Dantotsu, Murder y Regent. Se registró 2,00 a 2,75 adultos vivos promedio que fue estadísticamente similar entre estos productos, al primer día de evaluación. Al día 7 del control, los tratamientos que sobresalieron fueron Dantotsu y Murder. Se registró 0,75 a 1,25 adultos vivos promedio, que fue estadísticamente similar entre estos productos, al primer día de evaluación.

- Al día 14 del control, los tratamientos que sobresalieron fueron Dantotsu, Murder y Regent. Se registró 2,50 a 3,00 adultos vivos promedio que fue estadísticamente similar entre estos productos, al primer día de evaluación. También al día 14 del control, los tratamientos que sobresalieron fueron Dantotsu, Murder y Regent. No se registró adultos vivos al cuarto día de evaluación.

- La mayor eficiencia fue registrada en Dantotsu, con un $100 \%$ hasta los 14 días después de aplicar los tratamientos, seguido de Murder con un $100 \%$ de eficiencia hasta los 7 días después del control y $90 \%$ en 14 días.

\section{Referencias bibliográficas}

Alcázar, J. (1997). Producción de tubérculos semillas de papa. Manual de capacitación. CIP. Lima, Perú.

Basf (2011). Metaflubizona técnica. Disponible en: https://www.terralia.com/vademecum_de_productos_fitosanitarios_y_nutriciona les/view_trademark?book_id=1\&trademark_id=4842

Crissman, C. D.; Cole, Y. F. \& Carpio. (1994). Pesticide use and fram worker health in Ecuatorian potato production. American Journal on Agricultural Economics 76 (1 994):593-597.

Dale, W. (2013). Curso de Toxicología. Universidad Nacional Agraria La Molina, Escuela de postgrado, Entomología, s/p.

Lindo, G. E. (1993). Entomología agrícola. Universidad Nacional del Centro del Perú. Huancayo-Perú. 135p.

Nakahodo, N. J. (2003). Experimentación agrícola avanzada II. Escuela de post grado de la Universidad $\mathrm{Na}$ cional Agraria La Molina. s/p.

Paucarchuco, T. T. (2004). Plagas del cultivo de papa. Universidad Nacional del Centro del Perú. Huancayo-Perú. 145p.

Peralta, T. y Javier, G. (1980). Control integrado de las plagas de papa en el valle del Mantaro. Rev. Per. Ent. 23: 123-137.

Sánchez, V. G. y Vergara, C. C. (1991). Plagas del cultivo de papa. Universidad Nacional Agraria La Molina. Departamento de Entomología y Fitopatología. Lima - Perú. 86p.

Wille, T. J. (1956). Entomología agrícola del Perú. Segunda edición. Imp. Aramburú. Raygada Hnos. S.A. Junta de Sanidad Vegetal-Ministerio de Agricultura Lima-Perú. 543 p. 